\title{
Design Concepts for NiTiNOL Wires to Function as a Sensor
}

\author{
D. Josephine Selvarani Ruth ${ }^{1}$ (B)
}

Received: 10 November 2020 / Accepted: 6 January 2021 / Published online: 3 March 2021

(c) The Author(s) 2021

\begin{abstract}
Nickel Titanium Naval Ordinance Laboratory (NiTiNOL) is widely called as a shape memory alloy (SMA), a class of nonlinear smart material inherited with the functionally programmed property of varying electrical resistance during the transformation enabling to be positioned as a sensing element. The major challenge to instrument the SMA wires is to suppress the wires' nonlinearity by proper selection of two important factors. The first factor is influenced by the mechanical biasing element and the other is to identify the sensing current for the sensing device (SMA wires + biasing). This paper focuses on developing SMA wires for sensing in different orientation types and configurations by removing the non-linearity in the system's output by introducing inverse hysteresis to the wires through the passive mechanical element.
\end{abstract}

Keywords Shape memory alloy $\cdot$ Flexural element $\cdot$ Transformation hysteresis $\cdot$ Stress-strain $\cdot$ Electrical resistance

\section{List of Symbols}

$A_{f} \quad$ Austenitic transformation finish temperature at dynamic loading condition

$A_{s} \quad$ Austenitic transformation start temperature at static loading condition

$b_{A}, b_{M}$ Material constant

$C_{A} \quad$ The slope of austenitic transformation start and finish lines in the stress-temperature phase transformation diagram of shape memory alloy

$C_{M} \quad$ The slope of martensitic transformation start and finish lines in the stress-temperature phase transformation diagram of shape memory alloy

$A_{f} \quad$ Martensitic transformation finish temperature at dynamic loading condition

$A_{s} \quad$ Martensitic transformation start temperature at static loading condition

\section{Introduction}

A shape-memory alloy (SMA, smart metal, memory metal, memory alloy, muscle wire, smart alloy) is a class of smart material that has been receiving increasing attention in recent years owing to its ability "to remember" its original

D. Josephine Selvarani Ruth djsruth@gmail.com

1 Robert Bosch Centre for Cyber Physical Systems, Indian Institute of Science, Bangalore 560 012, India shape when subjected to thermal, mechanical or magnetic variations. This property can be used to generate motion or force in electromechanical devices and micro-machines. This material is a lightweight, solid-state alternative to conventional actuators such as hydraulic, pneumatic, and motor-based systems. Shape-memory alloys have applications in robotics and automotive, aerospace and biomedical industries.

These materials have the ability to undergo diffusionless martensite and austenite (solid-solid) transformations under different temperature and stress conditions. SMA exhibits a crystal transition between a high-temperature austenite phase and a low-temperature martensite phase. At low temperatures, an SMA exhibits a small Young's modulus and can be stretched easily; when heated, it contracts back to its original shape, overcoming almost twice its pulling force. SMA actuators are suitable to be adapted for applications that require flexible design/structure, and they are anticipated to play a pivotal role in the future due to their high energy density, mechanical simplicity, compactness, and clean operation. Although large force and displacement can be realized with these actuators, the effectiveness of the SMA actuators is typically hampered by two factors: hysteresis and bandwidth limitation. Transformation hysteresis is an important property of shape memory alloys which requires careful consideration during the material selection and while deciding on the technique to handle its nonlinearity so that its effect does not affect the system output; for technical applications under botheration. 
During the phase transformation, there are many inherent properties that undergo changes. One such important is the variation in the electrical resistance due to the contraction in the length. There are many literature reports about the sensing electrical resistance while in its actuation and the technique is self-sensing actuation. The concept of combining sensing and actuating features in the same device was first started by the work of Dosch et al. (1992) in piezoelectric cantilever. In shape memory alloy, self-sensing has been studied by various researchers like by Ikuta et al. (1988), Ma et al. (2004), Cui et al. (2010) and Lan et al. (2010a, b) employed mapping approach to determine the variation in ER as a feedback signal. Modeling approach, another technique of capturing ER has also been used in reports Majima et al. (2001), Dutta and Ghorbel (2005), Jayender et al. (2008), Liu et al. (2010), Furst et al. (2013). All these reports use sensing while in actuation and so they are selfsensing actuation.

The generic sensing principle is by the use of electrical resistance variation, this can be used as a sensor at a particular current. For a particular current, the SMA wire has a particular ER valve and on applying any external stimuli the ER varies accordingly.

Two factors of SMA (that contradict each other, are conflicting in the functionality to avail their usage) which kindled this investigation are the variation in electrical resistance, with which it can be used effectively for sensing while under actuation and, the presence of nonlinearity (in the alloy due to the transformation temperatures) in the actuator. This is the prime barrier to its lack of usage in commercial applications. The SMA-based system output must remain unaffected by the nonlinearity in SMA. Considering the above factors, an experimental analysis is conducted. The analysis reveals that the following two factors play an important role in the elimination/obscuring the effect of hysteresis in SMA: 'linearising current' of SMA and design/ selection of the mechanical biasing element.

The paper portrays three types of different force sensing, which gives the insight to customize the SMA wires in systems. The structure of the paper is as follows:

A. Mechanical design structure

2.1. Translational ( $x$-axis $)$

2.2. Longitudinal ( $Y$-axis $)$

2.3. Torsional

B. Mechanical inverse hysteresis

3.1. Passive

3.2. Active

C. Application in various field
4.1. Servoing

4.2. SDAS

4.3. Master system of a master-slave system

\section{Design of Force Measuring Devices}

The designing of inverse hysteresis involves two different approaches: one is passive and active. The practical construction of passive is done by using a cantilever beam and another by a passive spring. The active is by configuring two SMA wires (NiTINOL) in an antagonistic arrangement.

\section{Translational Force Measurement-Cantilever Element}

An experimental system chosen for the analysis consists of three components: a biasing element (that works in stressinduced martensite and for repetitive operation), SMA wire (the actuator), and proper mechanical assembly and package to support and protect these elements, respectively. The mechanical component is importantly the cantilever beam that acts as a bias element to the SMA wire and it is greatly necessary for repetitive operation. The biasing element can be passive such as dead mass/spring/cantilever beam and, the active element-an SMA element is connected in antagonism with its biasing element. The function of the biasing element is to bring back the SMA element to the product phase (martensitic phase) by directly applying stress on removing temperature thus making a direct transformation from austenite to detwinned martensite (by bypassing the twinned martensite). This direct transformation evolves only if it satisfies two indispensable conditions which are stated in the next section. In this test setup, a loop of SMA wire is connected between the free and fixed ends of the cantilever beam, at an angle. When the SMA wire in this setup undergoes the phase transformation it results in a wide hysteresis loop. Figure 1 depicts the mechanical system of the test set up.

\section{Longitudinal Force Measurement-Passive Spring}

This design uses a pair of SMA wires in synergistic configuration to each other to utilize the dual functionality of continuous sensing and actuation. This whole device is biased with a passive spring to achieve the sensing property and also for repetitive operation. An actuator/sensor is designed with shape memory alloy wires woven in simple open rectangular loops between supporting discs; such units are stacked along the central shaft to form the assembly. The structure looks like a parallel mechanism, used to accomplish large displacement and force since the design enhances 


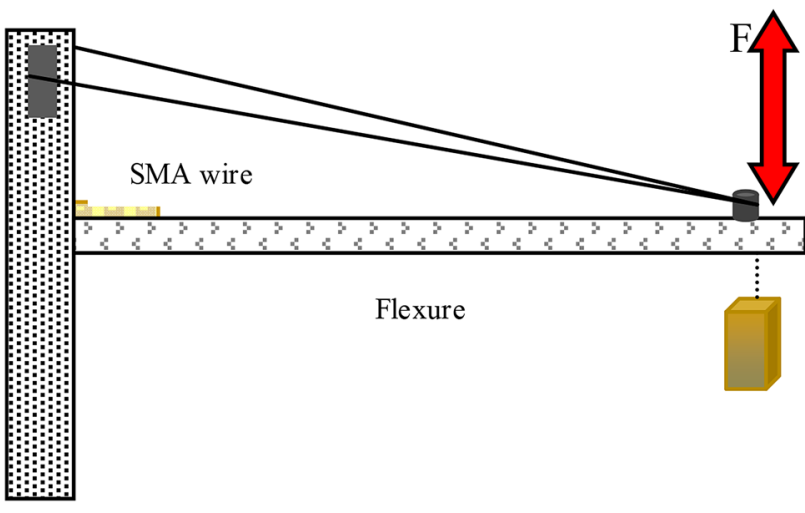

Fig. 1 SMA wire biased with a cantilever beam

the mechanical features (its displacement and force) of shape memory alloy.

This design does the function of both sensing and actuation leading to achieve haptics, suggested being named as sensaptics. The SMA sensaptic device consists of two units in synergetic configuration; two SMA wires are connected/ wound between pairs of discs (DA1 and DA2: outer and, DB1 and DB2: inner), in open rectangular loops. Disc DA1 is immovable while the other discs can slide along the central axis/shaft. The output of the device is referred from the position of disc DB2 with reference to the position of DA1. One SMA wire (SMA 1) is connected/wound between a pair of discs as follows: one end of a single strand of wire is attached to the disc DA1; the other end, runs through a hole in DB1, is then woven through DA2 and returns to DA1 through another radially placed hole in DB1. Similarly, another SMA wire (SMA 2) is attached/woven between the discs DB1 and DB2, passing through DA2. Discs DB1 and DA2 are attached/connected/coupled by non-flexible metal rods (see Fig. 2).

\section{Torsional Measurement}

The master is designed as a vertical structure with two columns connected by a horizontal shaft at a height of $14 \mathrm{~cm}$

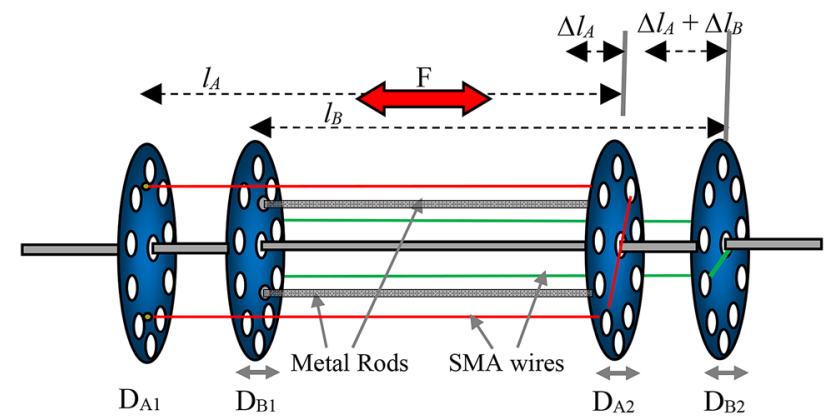

Fig. 2 SMA wire biased with passive spring from the base. The handle of the master is attached at the center of the shaft i.e., is similar to a joystick, an input device consisting of a stick that pivots on a base (the shaft) and reports its angle and direction to the device it is controlling (the slave). The one rotational degree of freedom of the joystick is driven by a pair of sensaptic SMA wires configured in antagonism. The joystick rotates about the centrally hinged point of the shaft whose movement is brought by the operator. This mechanism translates the force on the shaft to the strain as in Fig. 3.

\section{Design of Mechanical Inverse Hysteresis}

\section{Determination of Linear Operating Mechanism for Passive Biasing Element}

For linear sensing actuation, the range of stress should not destroy the elasticity $\left(E_{B}\right)$ of the beam. To make this system as in Fig. 4 work linear, the shape memory recovery should be in one of the three categories: free recovery, restrained recovery, and controlled recovery. In this design, the SMA employs restrained recovery where the martensitic residual strain of the SMA wire is restrained from being restored to its original length by the flexure. This restrains in the SMA wire should be offered by the flexure, which has to generate the deformation stress. The flexure gives constant stress which in combination with the SMA wire will produce the recovery stress so that it can obtain the ability to restrain. Figure 2a shows the forces related to the design $F_{1}$, $F_{2}, F_{3}$ (SMA forces $F_{s}$ ) are three different forces that are

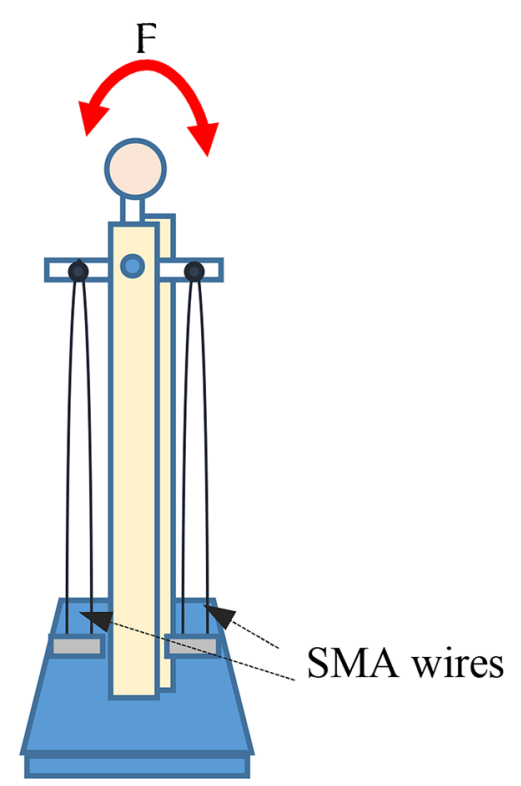

Fig. 3 SMA wire with active biased 
generated due to various currents $i_{1}, i_{2}, i_{3}$ correspondingly i.e., $F_{1}<F_{2}<F_{3}$ for $i_{1}<i_{2}<i_{3}$. For the sensing signal to be linear at a particular current of the SMA wire it has to satisfy two important conditions:

- The Young's modulus of the bias element should lie in between the forward and the reverse Young's modulus of SMA wire i.e., $\left(E_{r}<E_{B}<E_{f}\right)$

- The stress generated due to the force $\left(\sigma_{s}\right)$ should be equal to the stress offered by the flexure $\left(\sigma_{B}=\sigma_{s}\right)$. The current which equals the forces is selected to be $i_{S}$, the sensing current.

Song et al. (2003), Elahinia et al. (2004) and Furst et al. (2013) reported that stress-induced martensite phase transformations are one of the most important characteristics of SMA materials, the thermomechanical behavior of SMA can be described in terms of strain which made the basis for various stress-based models. The shape memory effect is caused by the phase transformation of the molecular structure between martensite and austenite. The range of stress where the bias element experiences when there is the transformation to austenite is given by Eq. (1):

$C_{A}\left(T-A_{f}\right)-\frac{\pi}{\left|b_{A}\right|} \leq \sigma_{f} \leq C_{A}\left(T-A_{s}\right)$.

For transformation to martensite, the stress applied should be within the range given by Eq. (2):

$C_{M}\left(T-M_{s}\right)-\frac{\pi}{\left|b_{M}\right|} \leq \sigma_{r} \leq C_{M}\left(T-M_{f}\right)$.

Under this operating condition, the system behaves in a linear manner. Figure 5 shows the linear region for the SMA wire and also the operating region for this linear sensing. The operating current can be at any point between A and B; the range of the sensor depends on the selection. At a point, A linearity starts and, offers a high range of force measurement. The range decreases after point $\mathrm{A}$ as $i_{S}$ nears $\mathrm{B}$, and at B nonlinearity enters.

Figure 5a depicts the force-resistance $(\mathrm{F}-\mathrm{R})$ characteristics of SMA during excitation with different magnitudes of current. From figure, it is understood that the hysteresis of $\mathrm{F}-\mathrm{R}$ relation reduces as the excitation current is reduced and, at $26 \mathrm{~mA}$ the response is basically linear.

Figure $5 \mathrm{~b}$ represents the tip displacement of the beam caused by SMA during the phase transformation.

\section{Determination of Linear Operating Mechanism for Active Biasing Element}

If the bias component is also an active element (i.e.,) another SMA element is connected in the antagonist configuration

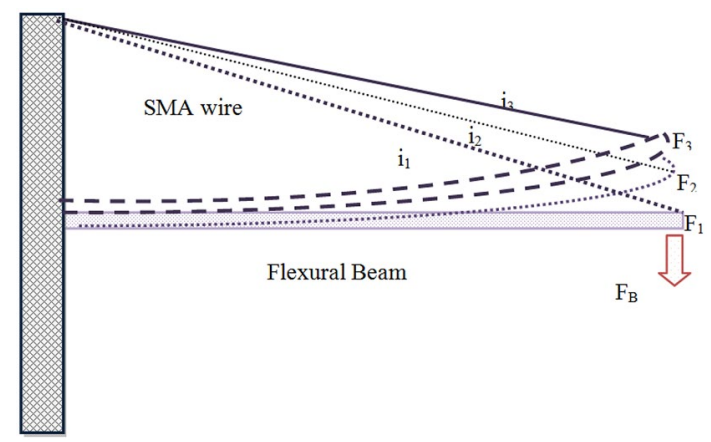

Fig. 4 Linear sensing mechanism-forces generated for different currents

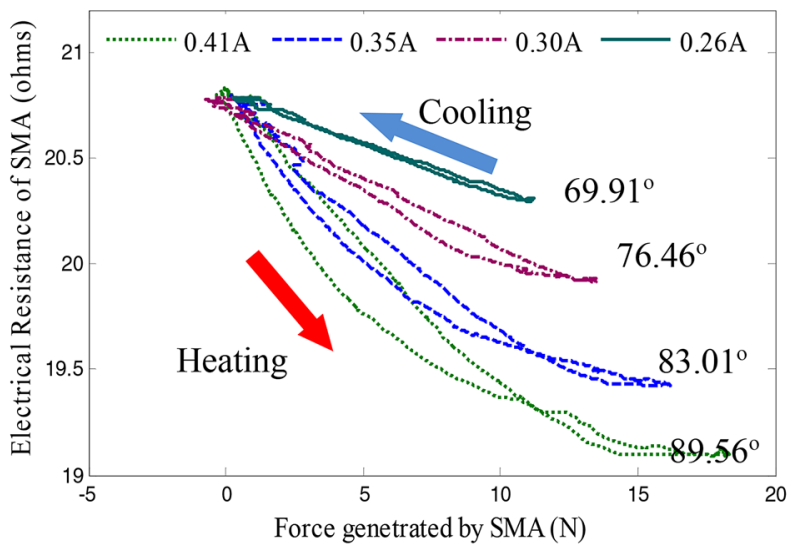

(a)

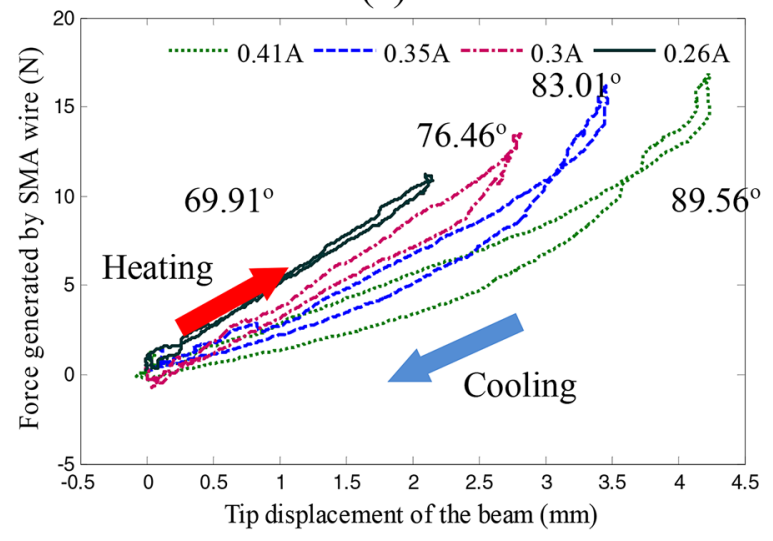

(b)

Fig. 5 Identification of the sensing current a force-electrical resistance characteristic, $\mathbf{b}$ displacement-force characteristic

and, if they are mainly operated in self-sensing actuation mode, then the conditions to be necessarily satisfied are as follows:

- In terms of the design whether the SMA as servo element adapted for linear or rotary movement, the force that is 
experienced at the point of application is the differential force (represented by suffix ' $D F$ ') generated by the wires, which should lie in between the forward and the reverse force generated in the SMA wire configuration i.e., $\left(\sigma_{r}<\sigma_{\mathrm{DF}}<\sigma_{f}\right)$.

- The stress generated due to the force of one forward transformation SMA wire $\left(\sigma_{\mathrm{fw}}\right)$ should be equal and opposite to the stress offered by the reverse transformation of the other SMA wire $\left(\sigma_{\mathrm{rv}}\right)$. The current which equals the forces is selected to be $i_{l}$, the linearising current and the stress-induced in the system is $\left(\sigma_{s}\right)$. This current is maintained in the SMA wires throughout the operation continuously and now, if one wire is contracted the stress generated by this actuated wire acts as an unknown mechanical force to the other wire and vice-versa, thus make the (self-)sensing to be linear and is also made to sense accurately and precisely.

While in operation the force applied on the system will be $\sigma_{s 1}+\sigma_{\text {fwo }}=\sigma_{s 2}-\sigma_{\text {rvo }}$.

In the linearized system $\sigma_{s 1}=\sigma_{s 2}$ and so, the stressinduced (compressive force) in the system by the forward operation $\sigma_{\text {fwo }}$ of one SMA will act as an unknown force (tensile force) on the other SMA, thereby enabling the sensing function to be effective, as the configuration is in the balanced state.

\section{Applications for the Proposed Devices}

\section{Force Servoing}

The flexure used in the system is a cantilever beam which acts as a bias, whereas the single SMA strand alone can be designed to behave as a sensor with any potential biasing element/system. This unique advantage allows SMA to be incorporated into any complex system/arrangement without consuming any space at a very low cost. A force servoing application-a dc servo motor operated by the command from the force sensor is considered and demonstrated. The desired force for the speed control system is set by the force applied to the cantilever beam and thereby the change in resistance of SMA acts as a command signal.

The speed of the motor is measured using an incremental encoder. A PID controller is used to control the system. The block diagram of the force servoing system is shown in Fig. 6. The dynamic sensing and speed control performance for dynamic input is presented in Fig. 7.

\section{Smart Pedal in the Driver Assistance System}

The design of the smart pedal module considers a pair of pedals, one for the accelerator and the other for the brake. The sensaptics is engaged via a mechanical linkage to the accelerator pedal, and integrated with the pedal spring in the car. When the accelerator pedal is pressed, the output disc of the sensaptics is displaced due to the pull of the linkage, with an associated variation in the electrical resistance of the SMA wire (SMA 2), which is the sensing signal of the pedal position sent to ECU. Likewise, when the ECU provides a control action, current flows through the SMA (SMA 1) wire thereby contracts the sensaptics. This provides a reflecting force/resistance to the foot of the driver on the pedal. Since this SMA-based sensaptics is used as a variable impedance actuator at any time, the driver can override the assistance system by applying more force on the pedal (Fig. 8).

The SMA-based sensaptics is featured with the functions of both sensing and actuation. The outer unit A (SMA 1) functions as the actuator and the inner unit B (SMA 2) is made to function as the force/displacement sensor. The SMA in the inner unit is continuously excited at a particular current (i.e., the inner unit is continuously at a minimal displaced state) such that it works perfectly linear for increasing and decreasing pedal forces. SMA is the part of the sensaptics as a non-linear element; hence to use the sensaptics as the pedal position sensor its linear operational region is determined experimentally.

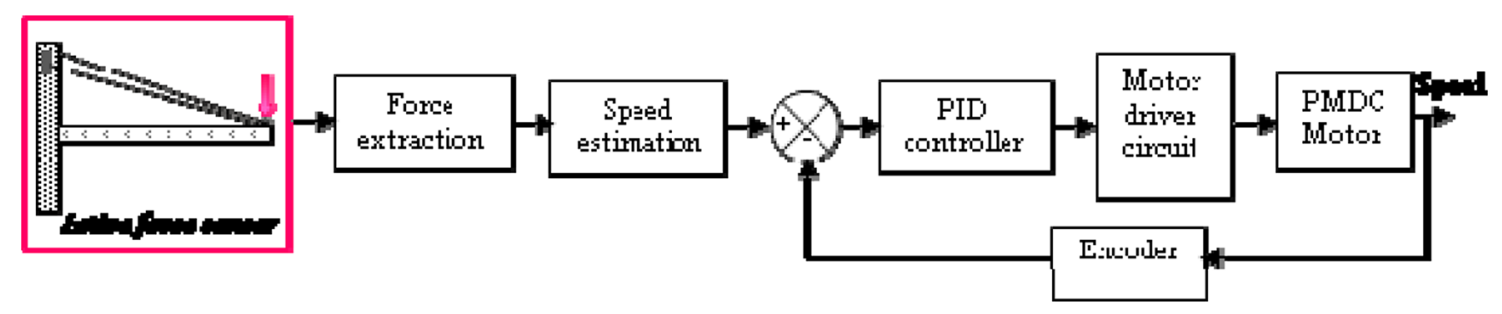

Fig. 6 Force sensor for speed control 
Fig. 7 Dynamic sensing and speed control for time-varying input a force to speed behaviour, $\mathbf{b}$ force application on the sensor, c corresponding speed variation in the dc servo motor (a)

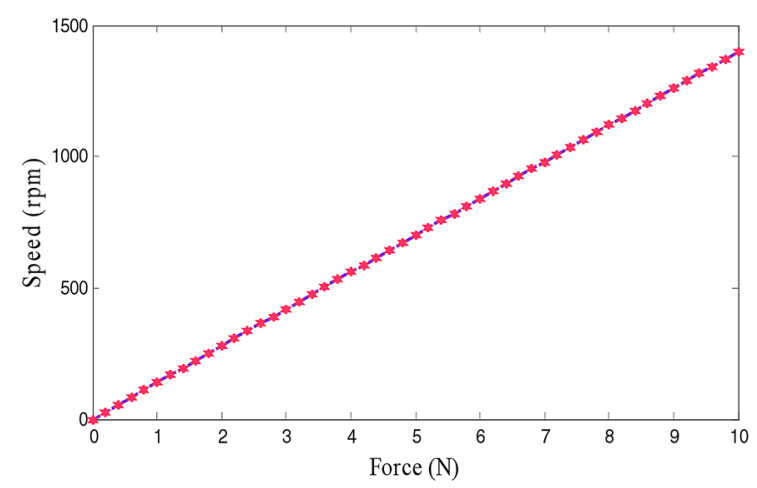

(a)

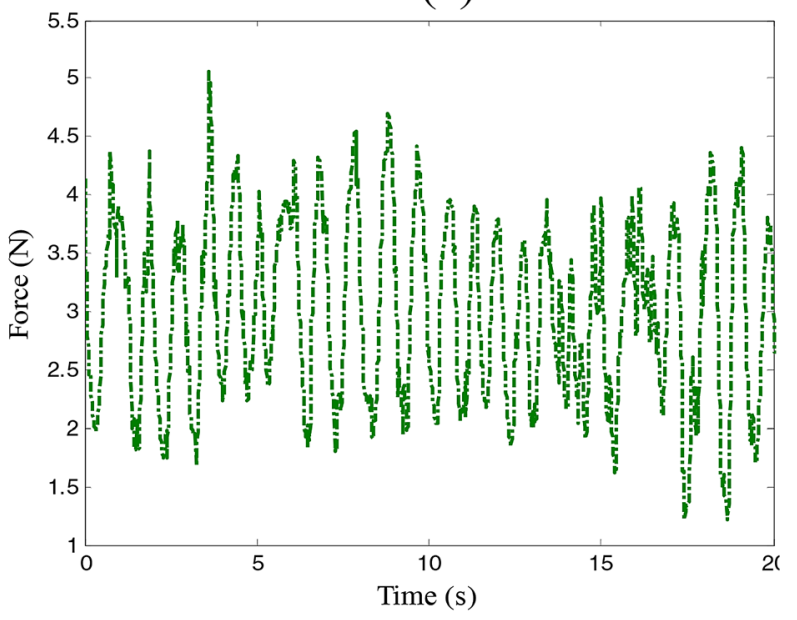

(b)

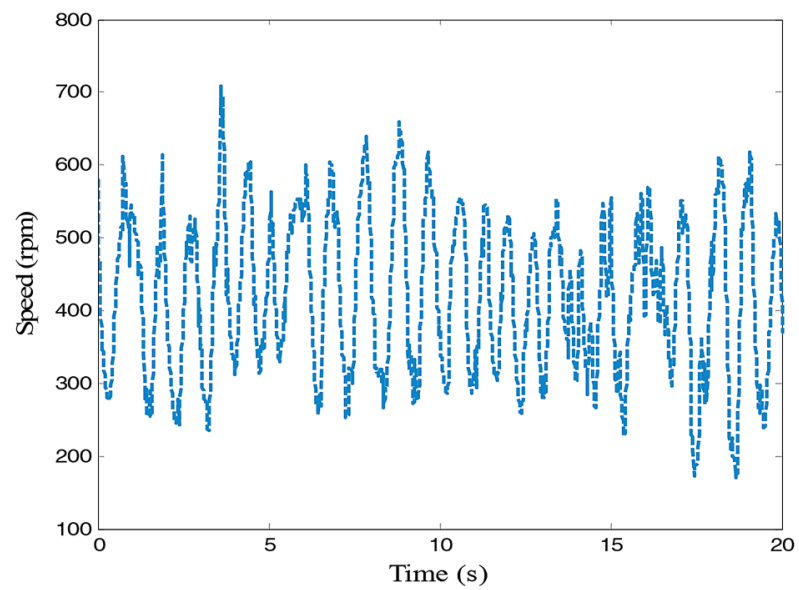

(c) 


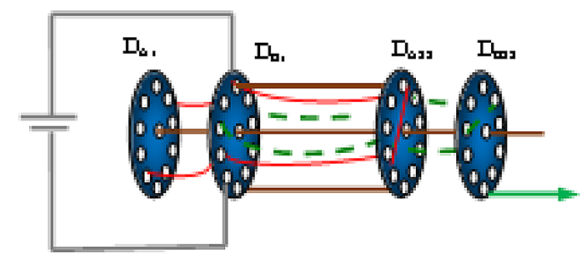

(a)

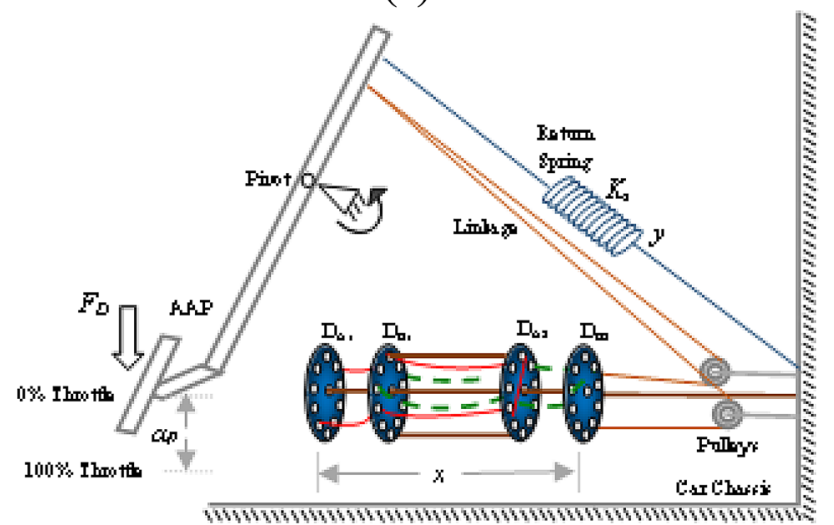

(b)

Fig. 8 Sensaptics in sensing mode a device in function, $\mathbf{b}$ device in AAP

\section{Design and Working of the Sensaptic Master-Slave System}

Bilateral control is one of the control techniques to transmit kinesthetic sensation bi-directionally. The proposed torsional measurement device is operated as a bilateral teleoperator, there are primarily two design goals which ensure a close coupling between the human operator and the remote environment. The first goal is that the slave manipulator should track the position of the master manipulator, and the second goal is that the environmental force acting on the slave when it contacts an article/detects impact in the remote environment, be accurately transmitted to the master.

In this haptic master-slave system, the SMA haptic master plays two roles: firstly it acts as the reference input device to the slave and secondly as a force feedback display device from the slave environment to the operator (Josephine Selvarani Ruth and Dhanalakshmi 2016a, b).

The SMA-based sensaptic arrangement is featured with the functions of both sensing and actuation/haptics. The sensaptic SMAs together function as the force/displacement sensor on continuous excitation at a particular current, to ensure linear operation. The appropriate excitation current $(0.25 \mathrm{~A})$ to the SMA is determined experimentally as in Fig. 9a for increasing and decreasing operator forces by examining the displacement of the manipulator and change in differential resistance; corresponding characteristics are plotted in Fig. 9b.

The linearity is enhanced due to the elimination of hysteresis in antagonism. Thus the antagonist wires perform bi-directional sensing. The SMA wires sense the rotational force of the operator, which induces a variation in the differential electrical resistance of the wires and is conveyed as the displacement (sensing signal), corresponding to the position of the master. This self-sensed differential electrical resistance variation in the SMA sensor is set as the reference input/information to operate the two-link robot. The position of the slave is affected by mapping the variation in the differential electrical resistance of the SMA wires to its co-ordinates. The dynamic behavior of the haptic master is established with this design; a typical response is shown in Fig. 9b.

\section{Conclusions}

Yes, the function of sensing is feasible with shape memory alloy wires. The results presented gives an insight that the SMA can be made to function as a linear sensing element. The transformation hysteresis in SMA wire has found the possibility of being eliminated using an appropriate linearising current for a particular passive or active mechanical element, which acts as a biasing element. The potential function of the designed sensors can be extended to various applications but not limited to bionics, rehabilitation devices, master-slave system, human-robot interaction, robotics, etc. 
Fig. 9 Input-output characteristic of SMA haptic master in sensing mode determination of activation current in SMA for linear sensing of the haptic master, $\mathbf{b}$ dynamic behavior of the haptic master

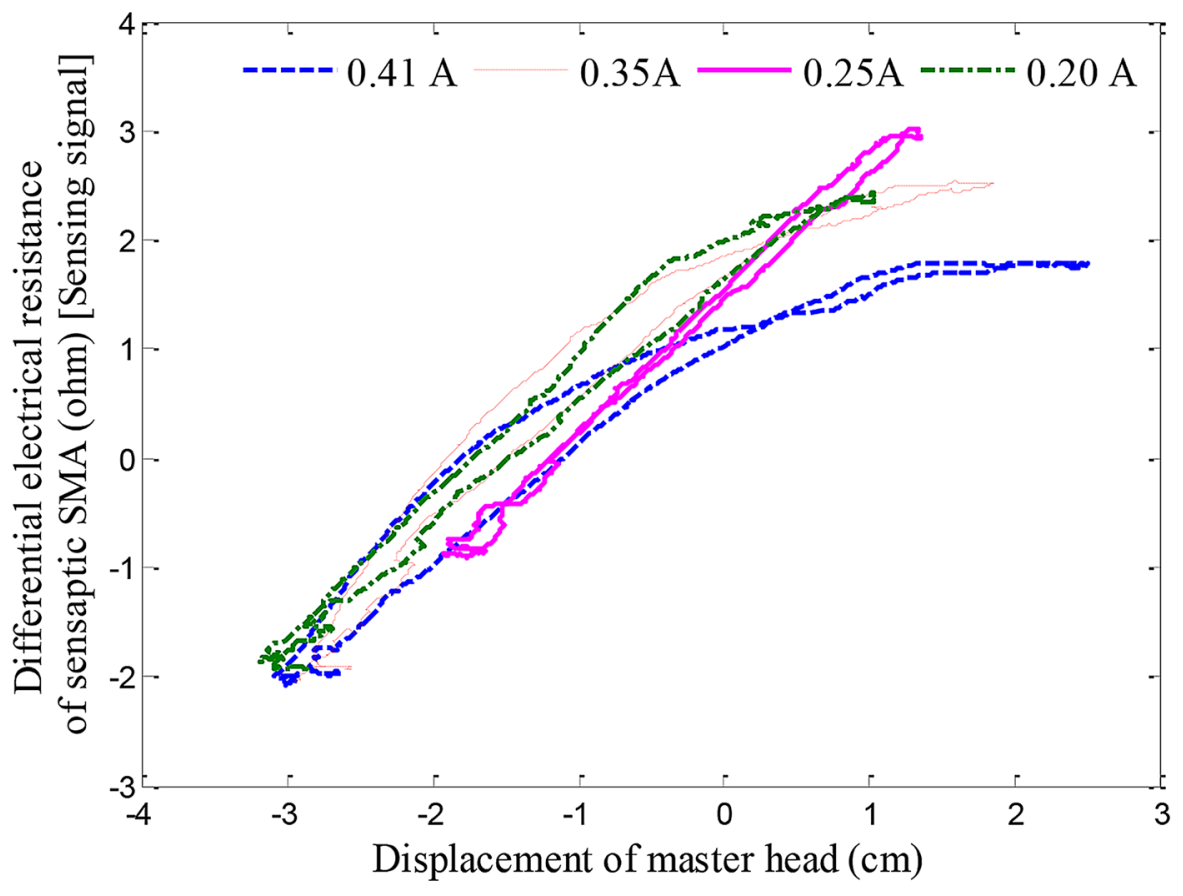

(a)

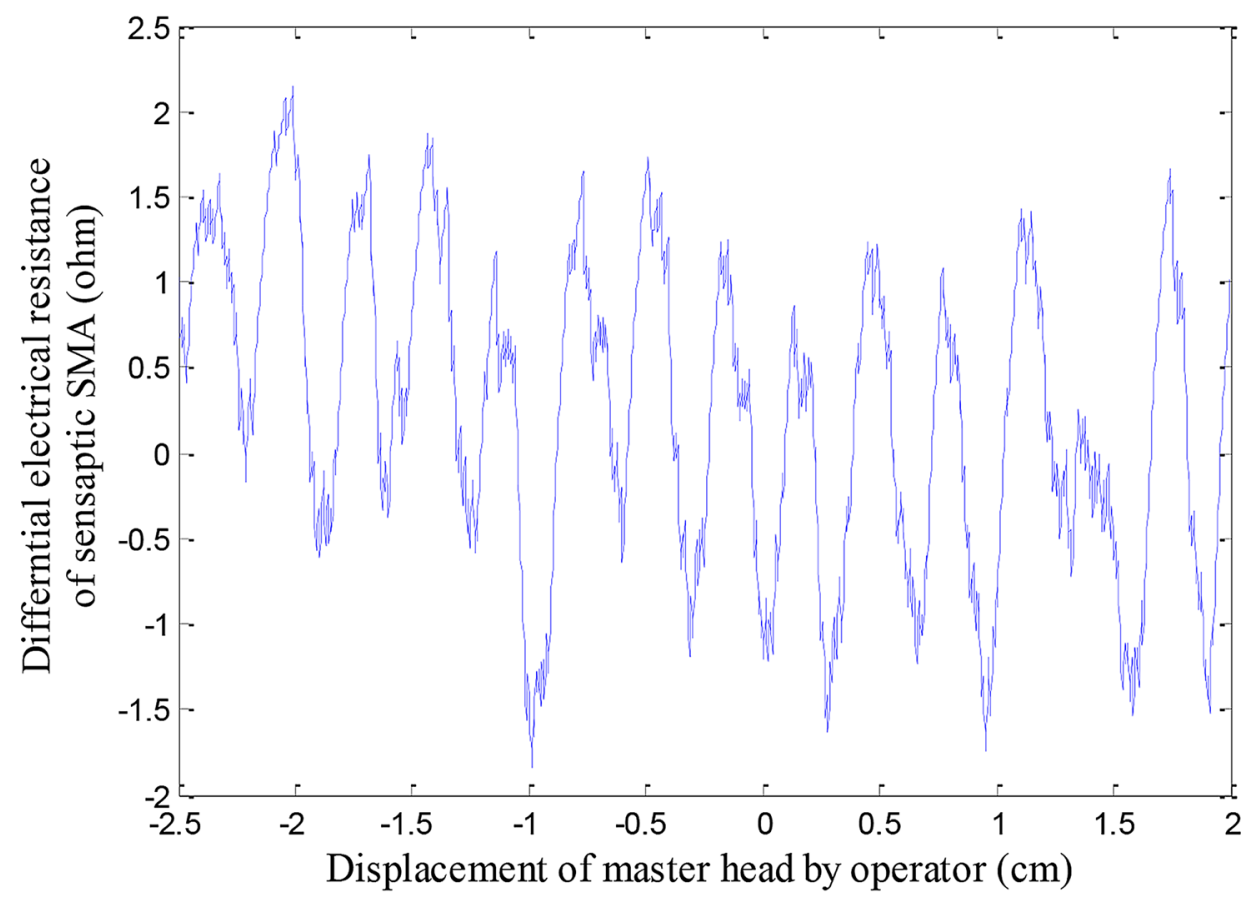

(b)

Open Access This article is licensed under a Creative Commons Attribution 4.0 International License, which permits use, sharing, adaptation, distribution and reproduction in any medium or format, as long as you give appropriate credit to the original author(s) and the source, provide a link to the Creative Commons licence, and indicate if changes were made. The images or other third party material in this article are included in the article's Creative Commons licence, unless indicated otherwise in a credit line to the material. If material is not included in the article's Creative Commons licence and your intended use is not permitted by statutory regulation or exceeds the permitted use, you will 
need to obtain permission directly from the copyright holder. To view a copy of this licence, visit http://creativecommons.org/licenses/by/4.0/.

\section{References}

Cui D, Song G, Li H (2010) Modeling of the electrical resistance of shape memory alloy wires. Smart Mater Struct 19:055019

Dosch JJ, Inman DJ, Garcia E (1992) A self-sensing piezoelectric actuator for collocated control. J Intell Mater Syst Struct 3:166-185

Dutta SM, Ghorbel FH (2005) Differential hysteresis modeling of a shape memory alloy wire actuator. IEEE ASME Trans Mechatron 10:189-197

Elahinia MH, Seigler TM, Leo DJ, Ahmadian M (2004) Nonlinear stress-based control of a rotary SMA-actuated manipulator. J Intell Mater Syst Struct 15:495-508

Furst SJ, John HC, Seelecke S (2013) Stress, strain and resistance behaviour of two opposing shape memory alloy actuator wires for resistance-based self-sensing applications. J Intell Mater Syst Struct 24(16): 1951-1968

Ikuta K, Tsukamoto M, Hirose S (1988) Shape Memory Alloy Servo Actuator System with Electric Resistance Feedback and Application For Active Endoscope. In: Procedings IEEE Robotics and Automation, Philadelphia, PA, USA, pp 427-430

Jayender J, Patel RV, Nikumb S, Ostojic M (2008) Modeling and control of shape memory alloy actuators. IEEE Trans Control Syst Technol 16:279-287

Josephine Selvarani Ruth D, Dhanalakshmi K (2016a) Shape memory alloy with bi-functionality in master system to control a slave. Sens Actuators A Phys 238:351-360
Josephine Selvarani Ruth D, Dhanalakshmi K (2016b) Shape memory alloy wire for force sensing. IEEE Sens J 17(4):967-975

Lan CC, Fan CH (2010a) An accurate self-sensing method for the control of shape memory alloy actuated flexures. Sens Actuators A $163: 323-332$

Lan CC, Fan CH (2010b) Investigation on Pretensioned Shape Memory Alloy Actuators for Force and Displacement Self-Sensing. In: Proceedings of The 2010 IEEE/RSJ International Conference on Intelligent Robots and System. Taipei, Taiwan, pp 3043-3048

Liu SH, Huang TS, Yen JY (2010) Tracking control of shape-memoryalloy actuators based on self-sensing feedback and inverse hysteresis compensation. Sensors 10:112-127

Ma N, Song G, Lee HJ (2004) Position control of shape memory alloy actuators with internal electrical resistance feedback using neural networks. Smart Mater Struct 13:777-783

Majima S, Kodama K, Hasegawa T (2001) Modeling of shape memory alloy actuator and tracking control system with the model. IEEE Trans Control Syst Technol 9:54-59

Song G, Chaudhry V, Batur C (2003) Precision tracking control of shape memory alloy actuators using neural networks and a sliding mode based robust controller. Smart Mater Struct 12:223-231

Publisher's Note Springer Nature remains neutral with regard to jurisdictional claims in published maps and institutional affiliations. 